\title{
Relatório de Gestão da Revista de Administração Contemporânea 2018-2021, e Farewell
}

The Journal of Contemporary Administration (RAC)'s 2018 - 2021

Managerial Report and a Farewell

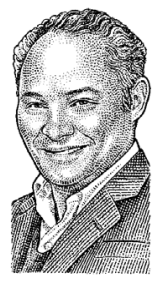

Wesley Mendes-da-Silva*1,2 두

Este é o último editorial que escrevo como editorchefe (EIC) da nossa Revista de Administração Contemporânea ( $R A C$ ), em cumprimento a um mandato voluntário pro bono com duração definida (2018-2021). Nesta oportunidade eu devo agradecer e reconhecer o trabalho profissional de algumas pessoas com as quais tive a grata oportunidade de trabalhar, e aprender, durante esses três últimos anos. Ao despedir-me desse papel, aproveito para deixar um breve registro resumido do trabalho realizado no dia a dia entre junho de 2018 e maio de 2021, em nome da boa prática de governança editorial de informar (Bandi \& Patton, 2019; Kumar \& Zattoni, 2019).

Cônscio dos deveres e responsabilidades inerentes ao papel de editor-chefe (Aguinis \& Vaschetto, 2011), no exercício do papel de EIC da RAC, busquei incansavelmente priorizar os interesses da comunidade brasileira de negócios nas inúmeras iniciativas da RAC ao longo desses anos. Para tanto, contei com a inestimável colaboração de pessoas de rara abnegaçáo, e de raro senso de dever profissional, entre essas destaco: a Editora de Casos para Ensino Paula Chimenti (CoppeAd/Universidade Federal do Rio de Janeiro/Brasil), que ampliou o trabalho iniciado por Victor Almeida (CoppeAd/Universidade Federal do Rio de Janeiro/Brasil),

* Autor Correspondente.

1. Fundação Getulio Vargas, Escola de Administração de Empresas de São Paulo, São Paulo, SP, Brasil. 2. Editor-chefe da RAC - Revista de Administração Contemporânea. e o Editor de Artigos Tutoriais e Dados Abertos Henrique Martins (Pontifícia Universidade Católica do Rio de Janeiro/Brasil). Agradeço aos Editores Associados Eduardo Flores (Universidade de São Paulo/Brasil), Emílio Arruda Filho (Universidade da Amazônia/Brasil), Fabio Caldieraro (Ebape/Fundação Getulio Vargas/Brasil), Gilnei L. Moura (Universidade Federal de Santa Maria/Brasil) e Paulo Matui. Meu especial reconhecimento aos Editores convidados para edições especiais: Airton Cardoso Cançado (Universidade Federal do Tocantins/Brasil), Ali Rostron (University of Liverpool/Reino Unido), Angela Paladino (University of Melbourne/Austrália), Aureliano Angel Bressan (Universidade Federal de Minas Gerais/Brasil), Benilde Oliveira (University of Minho/Portugal), Cristiana Fernandes De Muylder (Universidade FUMEC/Brasil), Cristiana Leal (Universidade do Minho/Portugal), Eduardo Schiehll (HEC Montréal/Canadá), Elin M. Oftedal (University of Stavanger/ Noruega), Emiliano A. Valdez (University of Connecticut/ Estados Unidos), Evelyn Lanka (EAESP/Fundação Getulio Vargas/Brasil), Giovanna Bertella (UiT The Arctic University of Norway/Noruega), Ines Branco-Illodo (University of Stirling/Reino Unido), Jairo Laser Procianoy (Universidade Federal do Rio Grande do Sul/Brasil), João Vinícius Carvalho (Universidade de São Paulo/Brasil), Luiz Ricardo Kabbach de

Como citar: Mendes-da-Silva, W. (2021). Relatório de gestão da Revista de Administração Contemporânea 2018-2021, e farewell. Revista de Administração Contemporânea, 25(6), e210100. https://doi.org/10.1590/1982-7849rac2021210100.por 
Castro (University of Florida/Estados Unidos), Małgorzata Grzegorczyk (University of Lodz/Polônia), Marcelo S. Perlin (Universidade Federal do Rio Grande do Sul/Brasil), María Luisa Esteban Salvador (University of Zaragoza/Espanha), Pallavi Singh (Sheffield Hallam University/ Reino Unido), Petra Molthan-Hill (Nottingham Trent University/Reino Unido), Ruby Roy Dholakia (University of Rhode Island/ Estados Unidos), Sanjay Lanka (EAESP/Fundação Getulio Vargas/Brasil).

Deixo registrado meu sincero e honesto agradecimento também às incansáveis pessoas que garantiram o funcionamento irretocável do escritório editorial da RAC: Kler Godoy, Nadia Machuca (que não mais pertence aos quadros da Anpad), e Simone Rafael. Agradeço, evidentemente, à Anpad pela confiança depositada e pela necessária independência permitida. Agradeço aos colegas que atuam em instituiçóes localizadas fora do Brasil, os quais aceitaram fazer parte da equipe editorial durante esse termo de gestão editorial: John L. Campbell (University of Georgia), Marcus Cunha Jr (University of Georgia/Estados Unidos), James Robert Moon Junior (Georgia Tech/Estados Unidos), Erica Piros Kovacs (Kelley School of Business/Estados Unidos), Julian Cardenas (Frei Universität/Alemanha), Elin Merethe Oftedal (University of Stavanger/Noruega).

Por fim, sabemos que poucos entendem a natureza do trabalho acadêmico, especialmente quando a doação de tempo no exercício de atividades profissionais em benefício da comunidade é a tônica, por isso preciso agradecer à minha família pela compreensão em momentos de ausência, para manter o processo editorial da RAC sob controle e em progresso.

\section{UM BREVE RESGATE}

Recebi, no final do primeiro semestre de 2018, a responsabilidade de conduzir os trabalhos atinentes ao processo editorial da RAC (Kimura, 2018). Tăo logo assumi o papel de EIC da RAC, busquei deixar clara a orientação que perseguiria no período de gestão 2018-2021: promoção da transparência, tanto do processo editorial quanto (e especialmente) da pesquisa publicada na revista, intencionando sair do campo das palavras, do discurso, indo para o campo da ação (Mendes-da-Silva, 2018a). Assim, desde o primeiro número deste termo de gestão da RAC, passamos a publicar artigos com dados abertos, de maneira a posicionar a revista na vanguarda pioneira da adoção de preceitos de ciência aberta no âmbito brasileiro na área de negócios. E, gradativamente, a política de ciência aberta foi refletida na revisão pelos pares, publicação de Artigos Tutoriais e de Cartas Executivas, conforme os editoriais detalhavam antecipadamente para o conhecimento da comunidade.
Em paralelo às atividades típicas de assunção de função, de modo a iniciar a execução de plano de trabalho apresentado à Anpad por ocasião do processo de recrutamento de EIC desta revista, procurei submeter a RAC aos indexadores dos quais se encontrava ausente. Uma série destes aceitou a revista sem maiores percalços. Contudo, aqueles de maior reconhecimento internacional, os conhecidos Scopus e Web of Science, apresentaram (após alguns meses de espera por uma resposta) uma diversidade de preocupaçóes que incluíam desde o fato de não termos uma versão em idioma inglês do website (impedindo o melhor entendimento das atividades da revista) até o layout dos documentos publicados.

De posse dessas preocupaçóes apresentadas pelos indexadores, iniciamos um trabalho concentrado em atender a tais demandas, e esse esforço nos conduziu a alcançar os seguintes avanços principais (alguns destes já registrados em relatório de gestão 2018-2019) (Mendes-da-Silva, 2019c), entre outros específicos que deixo de listar por motivo de espaço:

1. Construção de um novo e ampliado website da RAC na plataforma Open Journal System (OJS), permitindo: redução de custo, reduçáo do tempo de resposta, maior flexibilidade do processo de gestão da revista, autonomia da equipe editorial e melhor apresentação profissional da revista.

2. Renovação do Corpo Editorial Científico, de modo a reduzir endogenia em diferentes níveis.

3. Construção de versões em inglês e em espanhol de todo o conteúdo do website da RAC, especialmente em se tratando de suas novas políticas editoriais, de modo a permitir melhor e maior interlocuçáo com players internacionais, e.g., leitores, autores, agentes indexadores.

4. Redefinição e consolidação do escopo ímpar da RAC como revista de alcance internacional, e foco em temas de interesse regional.

5. Início dos procedimentos para manutenção da certificação do editor-chefe da RAC pelo Council of Science Editors (CSE).

6. Aumento da interlocução com colegas de outros países, por meio de convites para liderança de ediçôes especiais alinhadas com os Sustainable Development Goals da Organizaçáo das Naçóes Unidas (https://sdgs.un.org/goals recuperado em 11 de março de 2021). Isso permitiu que a RAC passasse a ser mais acessada por pesquisadores em países como Estados Unidos, Inglaterra, Alemanha, Portugal, Espanha e Noruega.

7. Criação da Seção de Artigos Tutoriais, uma nova classe de publicaçóes relevantes cujo foco é o método, 
de modo a permitir o aprendizado autônomo dos pesquisadores e a reduçáo de esforços redundantes em termos de execução da pesquisa, promovendo ainda o compartilhamento efetivo de ideias, em linha com práticas de open education, no âmbito dos princípios de open science (Martins, 2020; Mendes-da-Silva, 2018a, 2019b).

8. Criação e redefiniçâo de políticas editoriais com vistas à indexação e garantia da qualidade do processo editorial, conforme demanda o COPE - Committee on Publication Ethics.

9. Construção e adoção de um novo layout para os documentos publicados pela RAC (deixo aqui o registro do trabalho profissional da Sra. Kler Godoy), de modo a permitir a comunicação mais rápida, profissional e eficiente dos conteúdos (Mendes-da-Silva, 2019a). Nesse sentido, adicionamos, por exemplo: (a) JEL code (sistema de classificação desenvolvido pelo Journal of Economic Literature) de cada artigo; (b) número de convites realizados a revisores até que uma decisão editorial tenha sido viável; (c) adoção de resumos estruturados, anotação da contribuição de cada indivíduo participante da autoria do trabalho seguindo taxonomia CRediT; (d) nos Casos para Ensino, passamos a reportar quatro níveis de classificação (disciplina, temática, setor de atividade, região), de modo a facilitar a rápida identificação da audiência preferencial ao redor de cada Caso para Ensino.

10. Adoção da política de publicar todos os documentos em idioma inglês, de modo a estabelecer o posicionamento da revista náo pelo emprego do idioma, mas pelo escopo temático, colaborando para consolidação de uma identidade distintiva da RAC.

11. Estabelecimento da Editoria de Ciência Aberta, que se consolidou como vocacionada para gerir os interesses $\mathrm{da}$ revista com respeito às atividades rotineiras e estratégicas do processo editorial visando a consolidar a RAC como referência em open science.

12. Abertura gradual de dados, códigos, materiais e revisóes dos trabalhos publicados pela RAC, de natureza quantitativa ou qualitativa. Além dos argumentos tradicionais em favor de open science, intencionamos também uma externalidade que vimos como positiva: inibir a prática de salami science (Mendes-da-Silva \& Leal, 2021).

13. Adição de ambiente no website da RAC dedicado a organizar e disseminar material audiovisual relativo às atividades da revista, de modo a colaborar para o emprego crescente de mídias sociais em favor da maior capilaridade da revista e de suas publicaçóes (https://rac.anpad.org.br/index.php/rac/media recuperado em 11 de março de 2021).

14. Submissão e entrada da RAC em indexadores internacionais, de modo a aumentar a capilaridade dos trabalhos publicados. Por exemplo:
a. CiteFactor
b. COPE
c. Econpapers/Ideas (RePEc)
d. ERIHPlus
e. Europub
f. ICI Index Copernicus International
g. Latindex. Catálogo v2.0
h. MIAR
i. Redib
j. Sherpa/RoMEO
k. CARHUS Plus+

15. Submissóes sucessivas, até que a RAC fosse aceita (no final de 2020) para listagem no COPE, permitindo obter uma sinalização concreta para os indexadores e audiência da RAC acerca da qualidade e do rigor do processo editorial praticados na revista.

16. Lançamento (no volume 25 , número 4, 2021) da seção de Cartas Executivas, de modo a aumentar a interlocuçáo com o meio de praticantes da administração, e ao mesmo tempo fornecer relevantes informaçóes para a comunidade de pesquisadores acerca dos conteúdos que parecem prioritários para os indivíduos atuantes no front do cotidiano da administração.

\section{Estabelecimento de uma identidade distintiva e um escopo ímpar para a RAC}

A identidade da RAC tem sido marcada por um processo evolutivo, conforme narram Rossoni e Rosa (2020). Contudo, algo que parece factual é que a revista tem alcançado relativo sucesso no seu posicionamento como um periódico de destaque, sem que isso implique inexistência de trabalho a ser feito em termos de delineamento e consolidaçáo da identidade distintiva da revista, incluindo-se o seu escopo. Assim, de modo a náo deixar de realçar o acervo e a comunidade existente ao redor da RAC, neste ciclo editorial, buscamos atribuir fronteiras mais claras para o escopo e para a contribuição da revista para a sociedade. Como primeira atividade, no início do ciclo de gestão editorial, optei por investir algum esforço na definição de uma identidade visual que melhor caracterizasse a RAC, incluindo-se a construção de um website inteiramente novo, mais informativo e mais responsivo (Figura 1). 

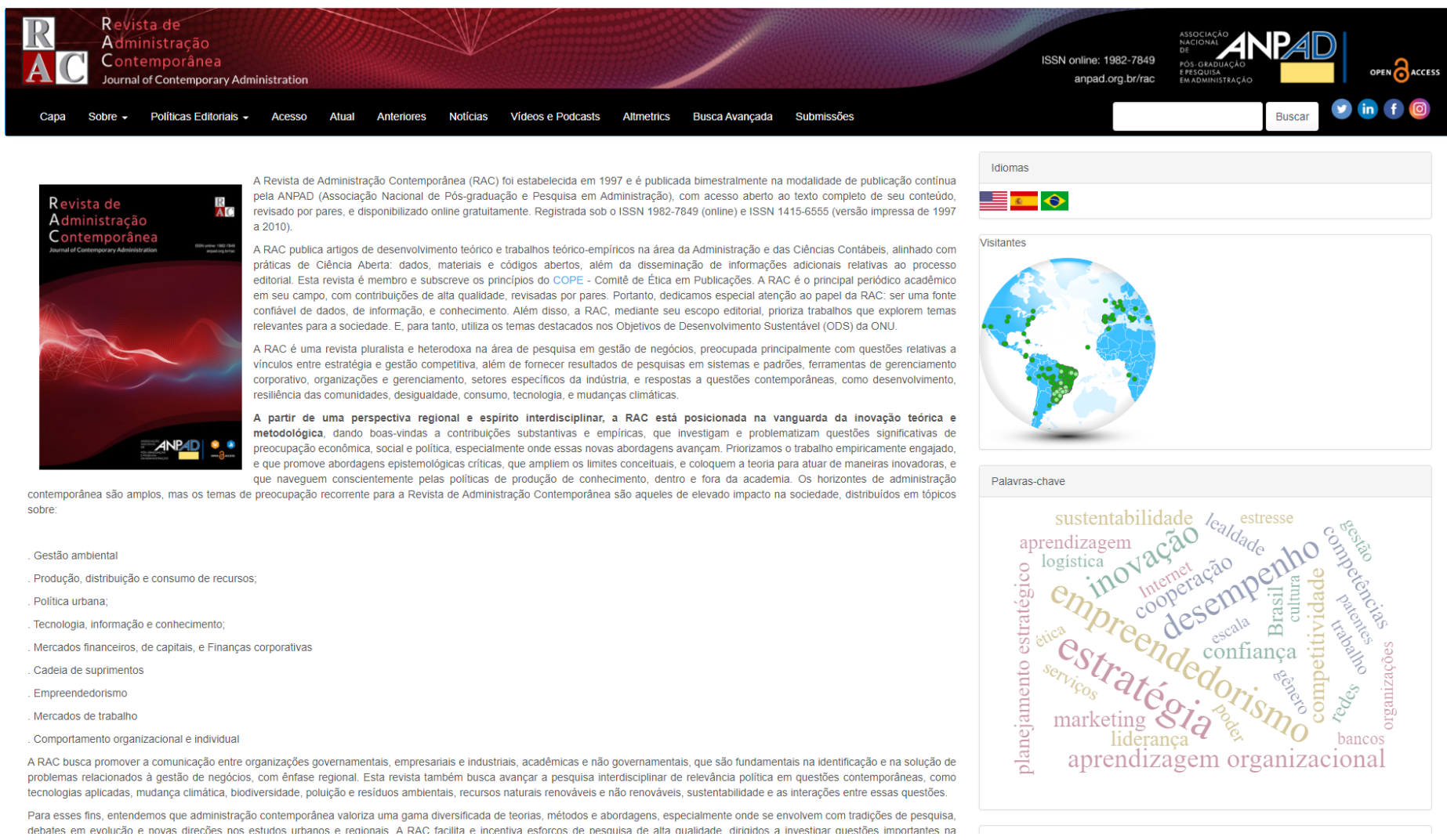

Figura 1. Vista do website da Revista de Administração Contemporânea-RAC.

Fonte: Website da RAC. Capturado em 9 de março de 2021. Esta figura está disponível em: http://doi.org/10.5281/zenodo.4591556.

Para além de aspectos estéticos e de apresentaçáo gráfica, houve especial preocupação em sinalizar para a comunidade o que a RAC intencionava publicar naquele momento, i.e., trabalhos alinhados com os princípios de open science, e que trouxessem contribuição para o debate ao redor da agenda de SDGs, e isso implicou a adoçáo de um conjunto de nove chamadas especiais planejadas, conforme segue:

1. Nudging and Choice Architecture (Leal, Oliveira, Branco-Illodo, \& Salvador, 2020): em andamento.

2. Research Methods in Qualitative Management Research (Lanka, Lanka, Ronstron, \& Singh, 2019): em andamento.

3. Insurance Industry (Carvalho, Flores, \& Valdez, 2020): em andamento.

4. Data Reuse: What New Information Can We Learn from Used Data? (Martins \& Perlin, 2020). Nenhum trabalho foi selecionado. Nós suspeitamos que mais esforço de divulgação e melhor informação da comunidade acerca do potencial de reuso de dados para a produção de novos trabalhos possam ser valiosos indutores de reuso de dados.
5. COVID-19's Impact on Businesses (Chimenti \& Marques, 2020): 16 Casos para Ensino dedicados às liçóes aprendidas com a pandemia de COVID-19 no Brasil, publicados no volume 25, número especial, 2021 (Marques, Chimenti, \& Mendes-da-Silva, 2021).

6. Business Models at the Crossroad of Responsible Innovation, Sustainability and Resilience (Oftedal, Bertella, Grzegorczyk, Hill, \& Lanka, 2020): publicada no volume 25, número 3, 2021.

7. Tutorial Articles: Guiding the Practice of Quantitative Research (Martins, 2019): publicada no volume 25, número 1, 2021.

8. Corporate Governance in Family Businesses (Mendesda-Silva, 2018b): publicada no volume 23, número 6, 2019. Registro aqui o agradecimento pela parceria estabelecida entre a RAC e o Instituto Brasileiro de Governança Corporativa - IBGC na realização dessa edição especial da RAC.

9. Technology Perspectives and Innovative Scenarios Applied in the Amazon Region (Arruda Filho, Muylder, Cançado, Dholakia, \& Paladino, 2019): publicada no volume 23, número 5, 2019. 
Ao longo desse termo de gestão editorial eu persegui a otimizaçáo da qualidade da revista, no âmbito da sua identidade distintiva. Nesse sentido, devo realçar que aspectos daí decorrentes, como internacionalização, não foram tratados como um fim per se, mas como consequência, externalidade. Isto é, a premissa foi: fazer o que precisa ser feito, e a revista científica naturalmente assumirá seu posicionamento no concerto dos periódicos de qualidade (no sentido de contribuição para a sociedade, para além da comunidade de pesquisadores).

Em que pese as práticas de open science, notadamente o compartilhamento de dados e materiais, não serem unanimidade entre autores e editores, players relevantes, como o Scielo e o COPE, já disseminam amplamente orientaçóes explícitas no sentido de promover gradual e irreversivelmente o compartilhamento público de dados, materiais, códigos de coleta e de análise de dados, pareceres emitidos pelos avaliadores e outras porçóes do processo editorial.
Tendo em vista esse movimento e o trabalho contínuo da RAC nesse sentido, os editores da RAC voluntariamente atenderam a convites e solicitaçóes de apresentaçóes acerca das práticas e aprendizados, como foi o caso da VIII Reunião Anual Scielo (Figura 2), de encontros da Anpad, de eventos científicos locais e de outros periódicos editados no Brasil cujos indivíduos editores estão interessados em adotar, e enfatizar, práticas de open science.

Contudo, o esforço envidado em direçáo a open science não vem desprovido da necessidade de alinhamento com práticas internacionais razoavelmente consolidadas. Nesse sentido, a RAC procurou estar em contato estreito com indivíduos e organismos de relevância na comunidade acadêmica, de maneira a acompanhar as práticas modernas e classificadas como as mais apropriadas. Uma ilustração disso foi a costumeira participação da RAC no Council of Science Editors - CSE Meeting 2019 (Figura 3), evento no qual pesquisadores, editores e publishers de diversas áreas de conhecimento encontram-se regularmente para o intercâmbio de ideias.

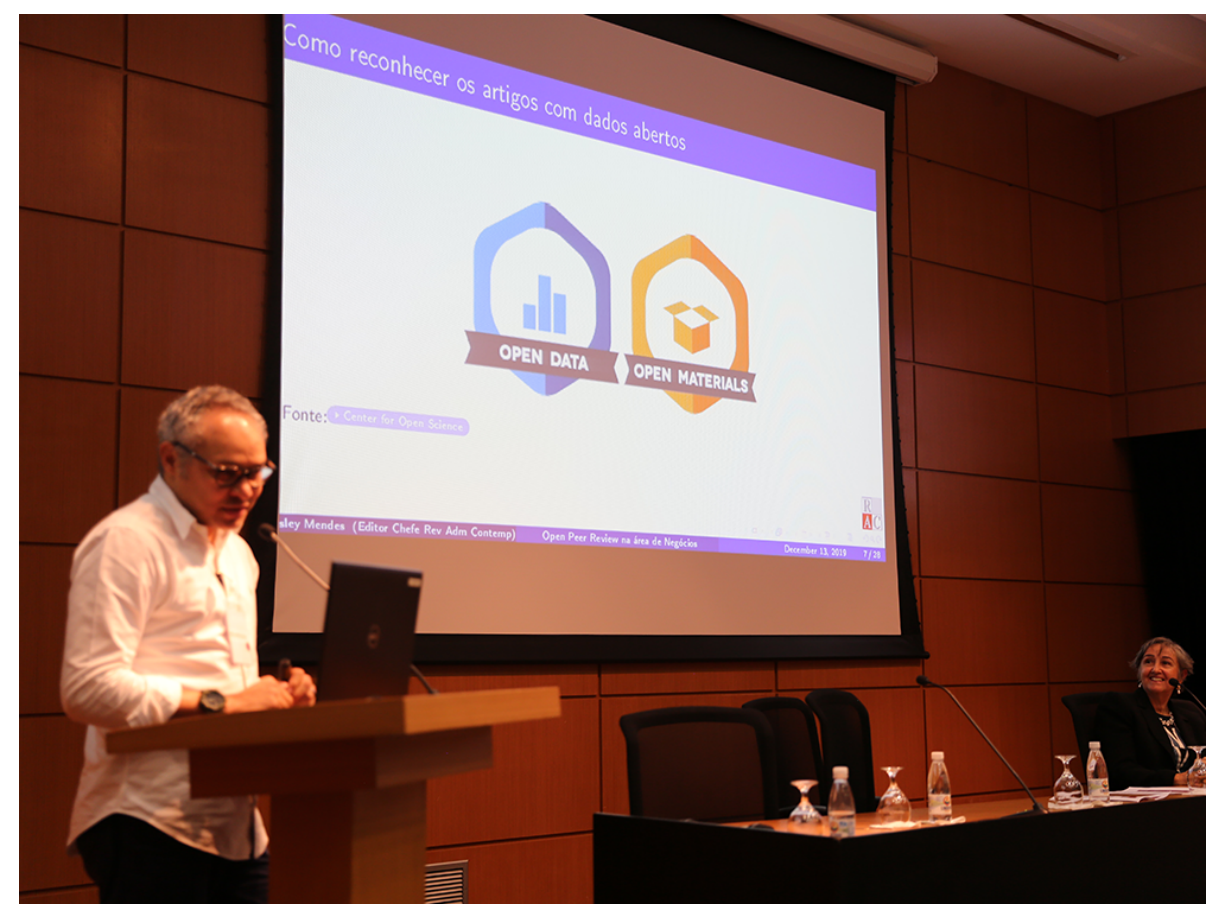

Figura 2. Apresentação da Revista de Administração Contemporânea - RAC na VIII Reunião Anual Scielo 2019, versando sobre práticas de ciência aberta adotadas pela RAC. Fonte: Galeria de fotos da VIII Reunião Anual Scielo (São Paulo, 13 de dezembro de 2019). Disponível em: http://eventos.scielo.org/viiireuniaoscielo/galeria-de-fotos/. A íntegra da apresentação realizada na oportunidade está disponível em: http://doi.org/10.5281/zenodo.3574011. 


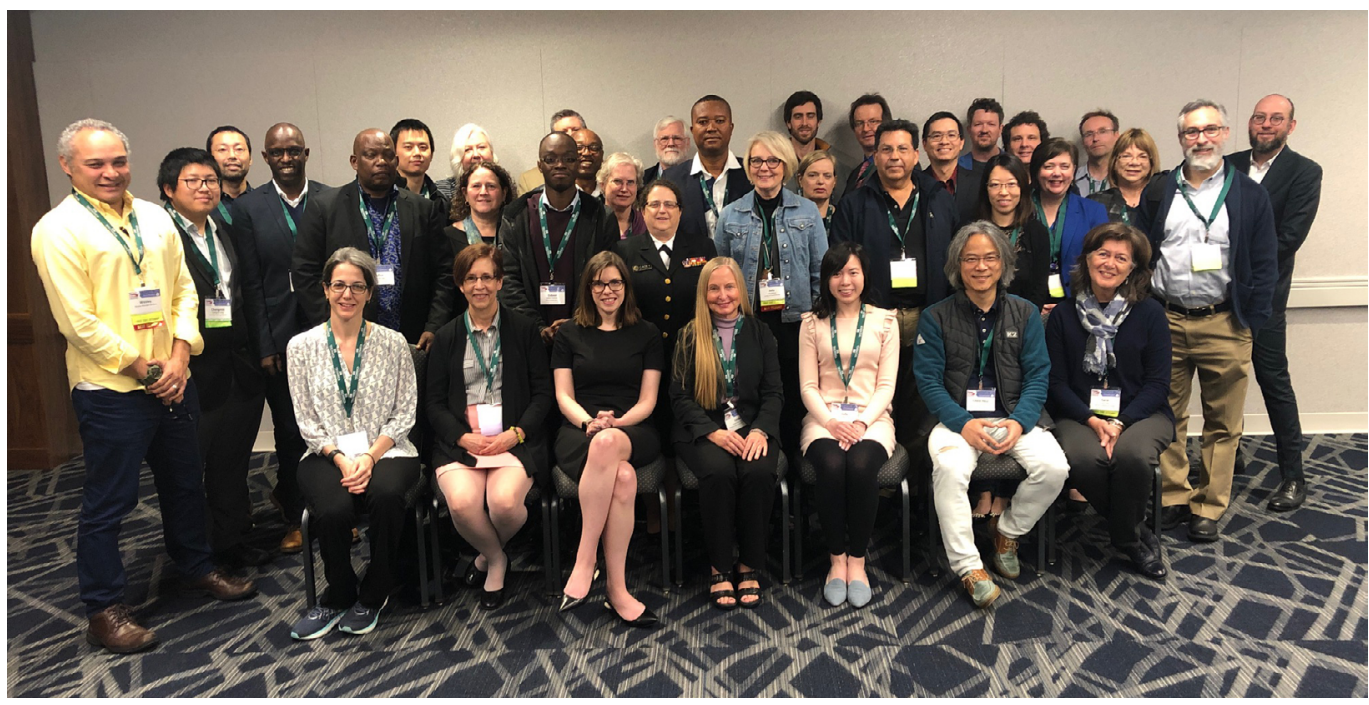

Figura 3. Grupo de participantes do Curso para Editores Científicos no Council of Science Editors 2019. Fonte: Gentileza de Christine G. Casey (Centers for Disease Control and Prevention), uma das colegas participantes do Council of Science Editors - CSE Meeting 2019, 4-5 de maio de 2019, em Columbus/Ohio.

Conforme as práticas de open science vão sendo consolidadas na RAC, e novos artigos forem publicados, paulatinamente a identidade da revista tomará contornos mais claros. Em consequência, tornar-se-á mais explícito o posicionamento editorial da revista, e sua contribuição para a sociedade, desempenhando o papel de fonte de conhecimento e de dados confiáveis. Em adição, pautas de elevada importância em open science, mas ainda carentes de desenvolvimento na comunidade, já começam a dar sinais de impacto por meio de algumas práticas e iniciativas da RAC.

A título de ilustração, ao passarmos a dar a opção a autores e revisores de tornar público o diálogo ao longo do processo de avaliação dos manuscritos publicados, felizmente uma parcela significativa dos trabalhos (Artigos e Casos para Ensino) apresenta as identidades dos indivíduos avaliadores, e especialmente o conteúdo na íntegra dos pareceres. Isso permite que novos avaliadores e novos autores possam apoiar-se nesse novo e valioso conteúdo para desenvolver suas habilidades. O reconhecimento do trabalho voluntário de avaliadores (Mendes-da-Silva, 2018c) é um ponto que merece especial cuidado por parte do editor, e tais práticas têm impactado positivamente os revisores atuantes na RAC (Figura 4).

Pedro Raffy Vartanian $\cdot 1^{\circ}$

Professor de Economia

$4 \mathrm{~h} \cdot($ ())

A atividade de parecerista de pesquisas científicas: no meio acadêmico, é comum que pesquisadores sejam convidados para a realização de pareceres de artigos científicos antes que os mesmos sejam publicados. No Brasil, especialmente, é uma atividade não remunerada e, normalmente, não reconhecida por ser uma atividade "invisivel". Os autores não sabem quem fez o parecer e o parecerista não sabe o nome dos autores. Isso garante que a avaliação seja meritocrática e totalmente despersonalizada. Sem prejudicar o anonimato prévio e a meritocracia, a RAC - Revista de Administração Contemporânea, que tem como Editor Wesley Mendes seguindo a tendência internacional de Open Science, adotou a prática de Open Peer Review, em que após a finalização do processo, os pareceristas (sob anuência), têm seu nome publicado no artigo nessa condição. Pela primeira vez após muitos anos e inúmeros pareceres para várias revistas, tive meu nome postado nessa condição no próprio artigo tutorial da RAC, o que também é uma novidade. "A GARCH tutorial with R". Parabenizo ao Editor, à equipe da RAC e aos autores do artigo pela publicação, disponível no link: https://Inkd.in/djnhmuT

Figura 4. Replicaçáo de post em rede social realizado por um revisor voluntário da Revista de Administração Contemporânea.

Fonte: Post realizado pelo Prof. Pedro Raffy Vartanian (ORCID: https://orcid.org/00000002-1492-7128) em uma rede social, acerca da OPR adotada pela RAC. 
É evidente que precisamos investir fortemente na qualificação dos revisores, sendo cogitado pela RAC, inclusive, fomentar um programa estruturado e formalizado (gratuito) de desenvolvimento e certificação de revisores, a exemplo do exitoso ACS Reviewer Lab, da American Chemical Society (2017). Todos nós sabemos da relevância de boas avaliaçốes, mas infelizmente ainda não temos um sistema que permita a estruturada instrução de indivíduos para fornecer bons pareceres; Precisamos (como comunidade) investir nessa agenda de profissionalização e de reconhecimento.

Quando olhamos especificamente para o acervo de artigos com dados abertos da RAC, constatamos que, dos 948 artigos publicados pela RAC entre janeiro de 1997 e dezembro de 2021, quase 60 têm dados e/ou materiais abertos. A política de dados abertos foi adotada em maio de 2018, a partir de quando foi paulatinamente incentivada, até que se tornou mandatória para trabalhos submetidos a partir do final de 2020.

Autores que desejarem conferir dados e/ou materiais abertos para artigos publicados pela RAC anteriormente a maio de 2018 podem fazê-lo sem maiores dificuldades, bastando seguir as orientaçóes que o escritório editorial da RAC compartilha para esse efeito. Um exemplo disso é um trabalho de autoria de Mendes-da-Silva, Onusic e Giglio (2013), que originalmente não tinha dados abertos, passando a tê-lo em 2018 (ver o ano de 2013 na Figura 5). Assim, há espaço para que uma expressiva parcela dos artigos já publicados pela RAC possa estar em linha com a moderna política de ciência aberta, dirigida a promover a produção e difusão acelerada de conhecimento (PLOS Media, 2021).

\section{UM POUCO A RESPEITO DE NÚMEROS}

Após quase 25 anos de trajetória, a RAC publicou uma diversidade de documentos que váo desde artigos científicos até Cartas Executivas (estas mais recentemente). Desde entáo, quase 950 artigos científicos inéditos passaram a estar disponíveis gratuitamente à sociedade, quase 60 deles com livre acesso aos dados e/ou materiais utilizados para o desenvolvimento da pesquisa (Figura 5), e que podem servir a novos pesquisadores. Conforme apresenta a Figura 5, desde 2018 a quantidade de artigos com dados e/ou materiais abertos vem crescendo rapidamente. Em contraste, a quantidade de artigos que não apresentam ao menos uma explicaçáo dos autores do trabalho para náo compartilhar os dados (dados secretos) vem sendo gradativamente reduzida, já que recentemente (final de 2020) a RAC, em atendimento a procedimentos adotados pelo Scielo, e em linha com o COPE, tornou mandatório o compartilhamento de dados.
Em determinadas situaçóes os dados podem acabar não sendo compartilhados, mas nesses casos o código de análise deve ser sempre compartilhado, caso tenha havido algum tipo de análise, mesmo que qualitativa. Um aspecto que merece investimento de atenção e desenvolvimento é a intensificação da política de dados e materiais abertos para estudos de natureza qualitativa (Aguinis \& Solarino, 2019; Moravcsik, 2014; Plakoyiannaki, Wei, \& Prashantham, 2019). Conforme destaco em editorial anterior (Mendesda-Silva, 2019b, p. 3): “Já é possível constatar, na comunidade internacional, iniciativas de classificação de periódicos conforme a reprodutibilidade típica da pesquisa publicada, por exemplo: https://replicationindex.com/tag/ replicability/".

Isto é, os benefícios advindos do compartilhamento de dados e materiais não são exclusividade de estudos quantitativos, até mesmo pelo motivo de a replicabilidade (a) constituir-se como um atributo que se aplica a qualquer estudo que implique a possibilidade de um indivíduo pesquisador interessar-se em repetir a pesquisa, independentemente do nível de replicação que esteja em consideração, ou (b) ser meio para assegurar requisitos mínimos para atestar a confiabilidade e a validade de resultados decorrentes de pesquisa qualitativa (Ethiraj, Gambardella, \& Helfat, 2016; Lecompte \& Goetz, 1982; Mendes-da-Silva, 2019b; Tsang \& Kwan, 1999). Contudo, não esqueçamos que, em última análise, o pesquisador é responsável pelo uso adequado e armazenamento (e/ou compartilhamento) de seus dados de pesquisa.

Em se tratando de interesse de outros países pelo conteúdo publicado na RAC, ao observarmos a Figura 6, verificamos que em termos de volume de acessos ao website da revista, Estados Unidos (ao redor de 8.500 acessos) e Portugal (ao redor de 6.500 acessos) têm sido os países que mais frequentemente aparecem, seguidos por Panamá, Moçambique, Reino Unido, Angola, México, Colômbia, Alemanha e Espanha.

Em que pese a RAC (ao menos ainda) não figurar nas famosas listas de periódicos considerados de maior qualidade, o que pessoalmente suponho que possa ocorrer muito em breve, é possível notar uma expressiva atenção recebida por revistas presentes nessas listas. A esse respeito, revistas listadas na Scopus, apenas no Brasil, já citaram trabalhos publicados na RAC mais de 1.600 vezes. A Figura 7 apresenta a frequência de citaçóes na Scopus, por país (porção à esquerda da Figura 7) e por periódico (porção à direita da Figura 7).

É notável a parcela de citações oriundas de Portugal, Estados Unidos, Espanha e Reino Unido. Mesmo assim, em termos de revistas, a RAC ainda é relativamente restrita a citaçôes advindas de revistas editadas no Brasil (com destaque para a Revista de Administração 
Mackenzie, Revista de Administração de Empresas e Revista de Administração Pública). Provavelmente, com a recente adoção de publicação do acervo integralmente (também) em idioma inglês, e com a política de open science em plena consolidação, é razoável esperar o aumento gradativo do total de citaçóes e da parcela oriunda de outros países.

Quando observadas as citações recebidas pela RAC originadas em outro indexador de relevância internacional, o Web of Science, foram encontrados 872 trabalhos publicados na RAC, os quais foram alvo de 3.321 citaçóes até 3 de março de 2021. A Tabela 2 apresenta a lista dos 50 documentos mais frequentemente citados no Web of Science até o início de março de 2021, sendo o texto intitulado "Construindo o conceito de competência", publicado no volume 5, número especial em 2001, o mais citado, com 107 citações.
Portanto, nas duas plataformas de contagem de citaçóes de alcance internacional, Scopus e Web of Science, a RAC evidencia um determinado número de citaçóes. Essa constatação, aliada aos avanços em diversos temas do processo editorial, permite assumir como razoável supor que em breve a revista estará presente nessas listas, e como resultado espera-se o aumento da quantidade e da qualidade dos trabalhos publicados pela revista muito em breve.

Ainda que não seja o motivo principal deste relatório de gestão editorial, convém registrar que não fez parte da alçada do EIC qualquer nível de decisão relativo à gestâo financeira decorrente das atividades editoriais, seja no planejamento orçamentário, seja na sua execução. Esse tema ficou restrito às Diretorias e Presidência da Anpad ao longo do termo deste mandato (maio/2018 a maio/2021).

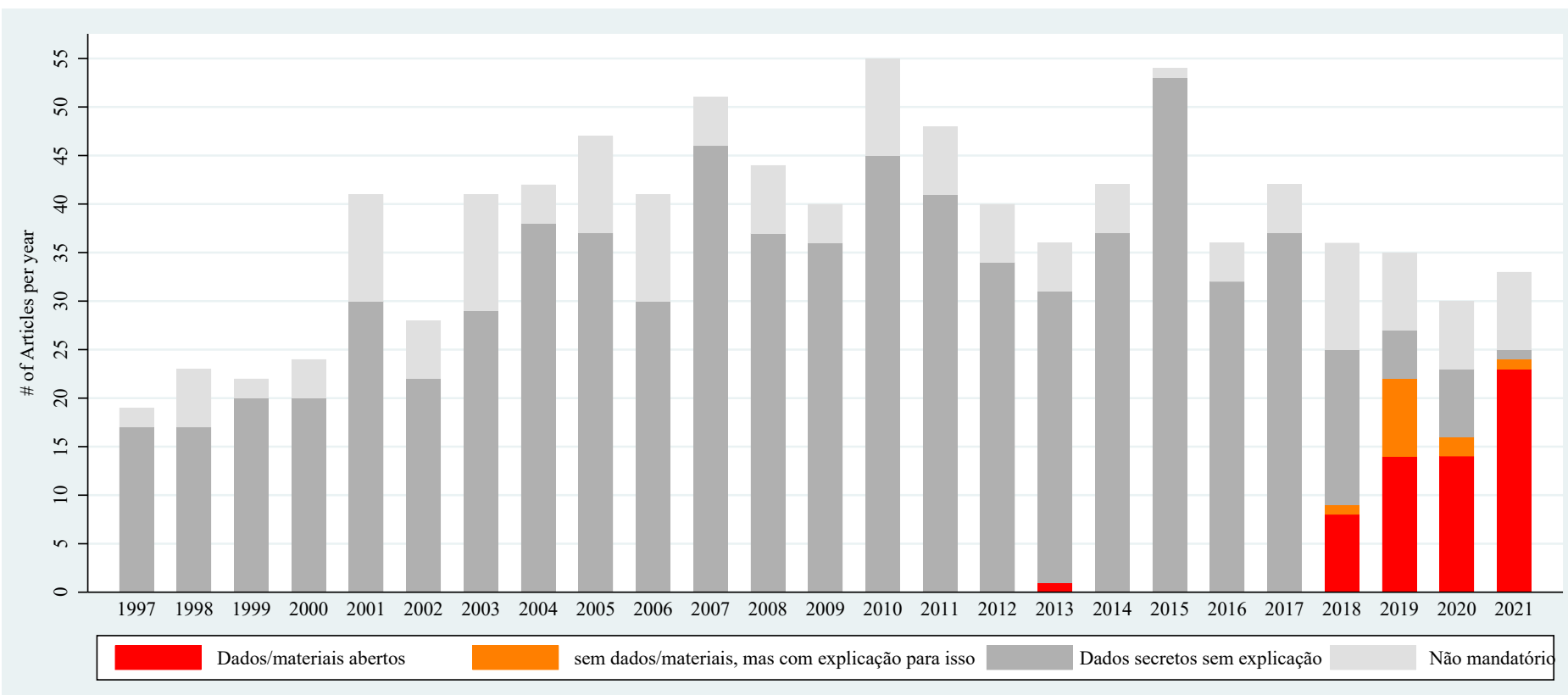

Figura 5. Evolução do número de artigos publicados pela $R A C$ e parcela de artigos com dados e/ou materiais abertos (1997-2021).

Fonte: Elaborado pelo autor com base em dados cedidos pelo escritório editorial da RAC. Nota: Esta figura apresenta a evoluçáo do número de artigos publicados pela RAC desde a sua fundação (1997), identificando a parcela de participação de artigos conforme o compartilhamento de dados e/ou materiais. A política de dados abertos iniciou-se em junho de 2018. Um artigo publicado em 2013 passou a ter dados abertos em 2018. O autor recorreu ao exame do conteúdo de cada um dos 948 artigos para classificá-los em cada uma das quatro categorias aqui reportadas. Esta figura está disponível em: http://doi.org/10.5281/zenodo.4591556. 


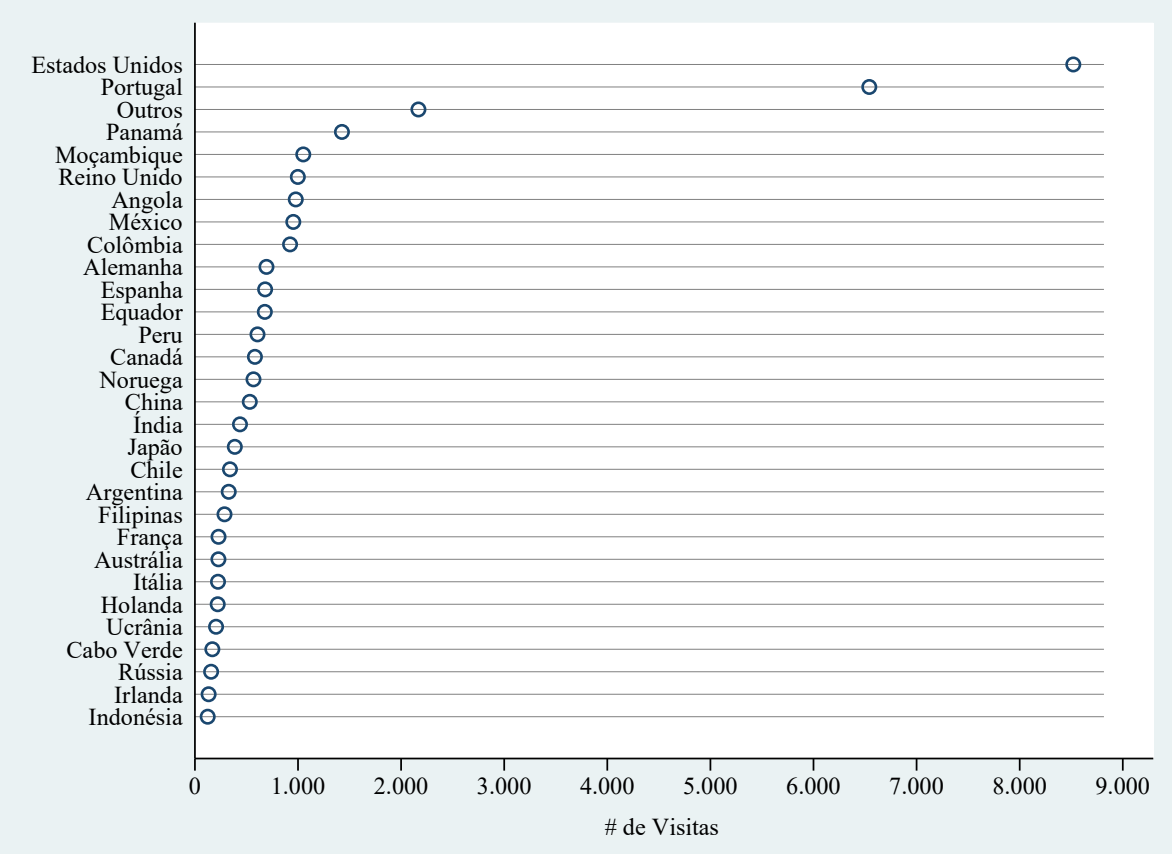

Figura 6. Lista dos 30 países que mais frequentemente acessaram o website da RAC (outubro/2018 a fevereiro/2021).

Fonte: Escritório editorial da Revista de Administração Contemporânea (RAC). Esta figura apresenta os 30 países mais frequentes em termos de origem de acessos ao website da RAC entre outubro/2018 e fevereiro/2021. Está ausente da figura o Brasil, com 475.190 acessos. O segundo país mais frequente são os Estados Unidos, com 8.520 acessos, destacando-se as cidades de Redmond/WA, Atlanta/GA, Chicago/IL e San Jose/CA, respectivamente. Além dos Estados Unidos, Portugal (6.541), Panamá (1.426) e Moçambique (1.051) são os países com ao menos 1.000 acessos. A categoria "outros" totaliza 2.168 acessos oriundos de países com menos de 124 acessos, quantidade constatada na Indonésia. Esta figura está disponível em: http://doi.org/10.5281/zenodo.4591556.
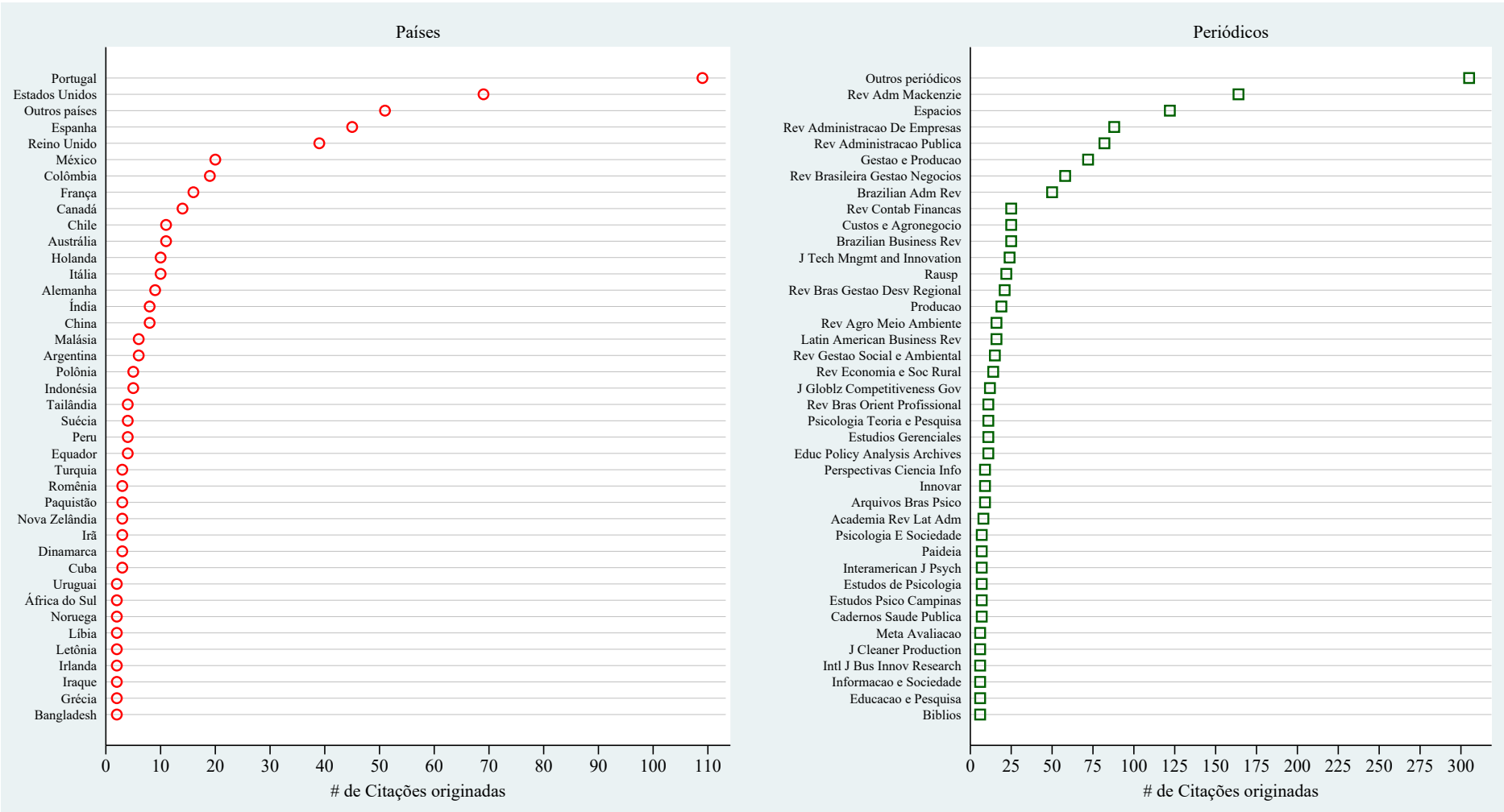

Figura 7. Citaçóes à Revista de Administração Contemporânea no indexador Scopus (conforme periódico e país † de origem da citação).

Fonte: Elaborado pelo autor a partir de dados gentilmente coletados pelo Prof. Luciano Rossoni na plataforma Scopus (em 3 de março de 2021). Esta figura apresenta, em ordem decrescente, as 40 fontes mais frequentes entre as citaçôes à RAC na plataforma Scopus. À esquerda estão os países mais frequentes, liderados por Portugal, com 109 citações, e Estados Unidos, com 69. † O Brasil, por motivo de melhor visualização da escala, está ausente da figura à direita, tendo em vista que 1.600 das citaçóes encontradas são oriundas desse país. À direita estão as revistas, lideradas pela Revista de Administração Mackenzie. A RAC passou a publicar seu acervo, integralmente, também em língua inglesa, considerados os trabalhos submetidos a partir de janeiro de 2020. Esta figura está disponível em: http://doi.org/10.5281/zenodo.459155. 
O processo editorial da RAC ocorre mediante a colaboração entre autores, revisores e editores, e ainda com a atuação do Escritório Editorial da RAC, fornecedores de serviços de revisão de texto e de suporte na preparação de formatos alternativos dos documentos para atender a critérios de indexadores. Esses players, em conjunto, acabam por determinar o quão veloz e criterioso é o trabalho que resulta nos documentos publicados. Assim, é um trabalho que não termina, está sempre em evolução. Portanto, é necessária a atenção especial nas diferentes fases do processo, que é caracterizado pelos indicadores. Adiante, na Tabela 1, estão resumidos os principais indicadores do trabalho desenvolvido na RAC entre o segundo semestre de 2019 e o segundo semestre de 2020. É possível verificar uma gradativa redução $(-54 \%)$ do ciclo editorial; isto se deve principalmente à presteza com a qual os pareceristas foram capazes de apresentar suas opinióes para os trabalhos submetidos, além da redução do número de trabalhos que seguem para peer review, de modo a poupar tempo e esforço tanto dos avaliadores quanto (e especialmente) dos autores.

Tabela 1. Caracterização do ritmo do processo editorial da Revista de Administração Contemporânea (2019/1-2020/2).

\begin{tabular}{lcccr}
\hline & \multicolumn{3}{c}{ Semestre } & \multicolumn{1}{c}{$\Delta \%$} \\
\cline { 2 - 4 } Indicador & $2019 / 1$ & $2020 / 1$ & 16,7 \\
\cline { 2 - 4 } Total de artigos submetidos & 150 & 167 & 175 & $-50,0$ \\
Artigos em avaliação & 100 & 63 & 50 & 0,0 \\
Artigos aceitos e publicados & 12 & 15 & 12 & 275,0 \\
Artigos aceitos a serem publicados & 8 & 10 & 30 & $-54,1$ \\
\hline Tempo médio (dias) entre submissão e publicação & 133 & 78 & 61 & \\
\hline
\end{tabular}

Nota. Fonte: Escritório Editorial da Revista de Administração Contemporânea - RAC, refletindo a posiçâo do sistema de gestão editorial em 11 de março de 2021 . A última coluna mostra a variaçáo percentual entre o primeiro e o último período.

\section{DESAFIOS VINDOUROS À RAC}

Em que pese haver indicaçóes de que a permanência por períodos menos curtos na editoria de um periódico pode induzir melhor aproveitamento dos aprendizados adquiridos pelo editor, em favor do processo editorial da revista, pessoalmente, vejo que os ganhos líquidos de permanecer por mais tempo na editoria podem ser negativos, pois permanecer por mais tempo pode ao mesmo tempo: deixar de aproveitar a energia que novos (não necessariamente os mais jovens) indivíduos editores podem trazer, deixar de aproveitar novas ideias e concepçóes latentes na comunidade, induzir acomodação no ritmo de trabalho do editor e equipe editorial e impedir que o editor possa refletir sua experiência em outras esferas da comunidade que guardem sinergia com a experiência adquirida na gestão do processo editorial.

Por esse motivo, é inegável que mesmo que a revista tenha avançado em alguns tópicos, o processo de desenvolvimento náo tem como deixar de ser permanente, há e haverá sempre muito a ser feito, especialmente quando os recursos são escassos. Portanto, desafios são parte da agenda de trabalho do editor. Com a intenção de deixar uma pequena contribuição para a agenda de trabalho vindoura, seguem aspectos que julgo merecedores de especial atençáo, tendo em vista a missão da RAC como patrimônio da comunidade, construído a partir da colaboraçáo de editores e autores (deixo aqui o registro de reconhecimento pelo trabalho desenvolvido pelos editores predecessores à frente da RAC desde a sua fundação: Clóvis Machado-da-Silva 1997-2001, César Gonçalves Neto - 2001-2002, Tomás de Aquino Guimarães - 2002-2005, Rogério Hermida Quintella - 2006-2012 e Herbert Kimura - 2012-2018).

Desenvolvimento da qualidade e da celeridade dos pareces recebidos: Os avaliadores anônimos constituem parte essencial do processo editorial, e como tal devem receber especial atenção no processo editorial. A RAC conta com centenas de pessoas que doam seu tempo para esse papel. Mas é necessário diversificar, ampliar, qualificar e reconhecer o trabalho dessas pessoas cada vez mais raras.

- Consolidação da política de ciência aberta: A iniciativa de promoção da transparência induzida em meados de 2018 constitui-se como chave para o crescimento de consolidação da RAC, para que a revista esteja em linha com as modernas práticas editoriais adotadas ao redor do mundo.

- Exploraçáo do potencial de inserção (de maneira profissional e estruturada) da revista via mídias sociais: A par da necessidade de impactar a sociedade, é inegável a conveniência de estratégias e práticas profissionalizadas (não aleatórias ou casuísticas) de 
emprego de mídias sociais, e isso implica alocação de recursos (não apenas financeiros).

Consolidação da revista como fonte de dados críveis: Uma externalidade positiva da política de open science é o acúmulo de material compartilhado pelos autores. Em decorrência disso, naturalmente a revista passa a desempenhar o papel de fonte de dados para realização de pesquisas. Isso pode ocasionar diversos impactos positivos, e.g.: reduçáo dos custos de realização de novas pesquisas, inserção de novos pesquisadores que experimentam dificuldades para acesso a dados, atraçáo de novos indivíduos para a audiência da RAC (como a imprensa de negócios).

- Estabelecimento de canais consistentes de interlocução com a sociedade: Colocadas em conjunto política de dados e materiais abertos, uso de mídias sociais, diversificaçáo da base de avaliadores, atração de autores de novos contextos, passa a ser viável considerar a intenção de tornar a RAC um elo entre a comunidade de pesquisadores e a sociedade.

- Atração de submissóes oriundas de outros países: Deve ser naturalmente impulsionada quando em breve a RAC for listada nos indexadores de maior relevância internacional, após esforços de interlocução com os indexadores, e.g., listagem no COPE, versão em inglês do site, publicação integral em língua inglesa, chamadas especiais lideradas por colegas atuantes em outros países, entre outras iniciativas.

Consolidação de sua identidade editorial: Não parece razoável, ao menos para este editor, estabelecer a identidade de um periódico científico com base no idioma em que os trabalhos são publicados. Em vez disso, cabe buscar a ênfase nos delimitadores do escopo e da missão da revista. Ao longo deste ciclo de gestáo editorial foram envidados esforços para adotar um escopo mais claramente contributivo para a comunidade, até mesmo para que fossem satisfeitas demandas de agentes indexadores e órgãos de certificação de alcance e reputação internacionais com os quais a RAC passou a buscar interlocuçáo mais estreita, de modo a realçar o seu papel.

Investir esforço em identificar meios alternativos de sustentabilidade financeira: Tendo em vista as crescentes restriçóes financeiras que limitam o crescimento e a profissionalização da revista, ainda que os esforços para vencer dificuldades sejam louváveis, é necessário refletir acerca de meios de diversificar o risco da sustentação financeira da RAC, e.g., permitir a percepção de doaçóes de indivíduos e organizaçóes, sem que isso implique comprometer a lisura do processo editorial.

Estrutura segue estratégia: A par de desafios que se apresentem à RAC, deixo a sugestáo para que náo somente o destino da revista seja claramente demarcado, mas também que em função dele haja um especial cuidado para estruturar a revista em termos de recursos de diversas espécies.

Espero que saiamos deste período de pandemia com saúde e com aprendizados para nossas vidas. Muito obrigado à comunidade pela confiança e pela oportunidade de aprender. Até breve, e sorte à nova editoria da RAC!

Tabela 2. Artigos publicados na RAC mais frequentemente citados na Web of Science (posição em 3 de março de 2021).

\begin{tabular}{|c|c|c|c|}
\hline N. ${ }^{\circ}$ & Título & URL para acesso direto ao artigo & $\begin{array}{l}\text { \# de } \\
\text { citaçôes }\end{array}$ \\
\hline 1 & Construindo o conceito de competência & https://doi.org/10.1590/S1415-65552001000500010 & 107 \\
\hline 2 & $\begin{array}{l}\text { Content analysis as a qualitative data analysis technique in the field of } \\
\text { administration: potentials and challenges }\end{array}$ & https://doi.org/10.1590/S1415-65552011000400010 & 69 \\
\hline 3 & Research process and bibliometric analysis: bank service quality assessment & https://doi.org/10.1590/S1415-65552013000300005 & 39 \\
\hline 4 & $\begin{array}{l}\text { Produção científica em administração de empresas: provocaçóes, insinuações e } \\
\text { contribuiçóes para um debate local }\end{array}$ & https://doi.org/10.1590/S1415-65551999000100009 & 34 \\
\hline 5 & What is a theoretical essay? & https://doi.org/10.1590/S1415-65552011000200010 & 33 \\
\hline 6 & $\begin{array}{l}\text { Patterns of productivity of Brazilian authors in accounting magazines and } \\
\text { congress: a study bibliometric }\end{array}$ & https://doi.org/10.1590/S1415-65552008000200011 & 25 \\
\hline 7 & The field of studies of inter-organizational cooperation networks in Brazil & https://doi.org/10.1590/S1415-65552010000300005 & 22 \\
\hline 8 & A tradição anglo-saxônica nos estudos organizacionais brasileiros & https://doi.org/10.1590/S1415-65552001000500005 & 21 \\
\hline 9 & $\begin{array}{l}\text { A bibliometric approach to Brazilian scientific production on corporate social } \\
\text { responsibility - CSR }\end{array}$ & https://doi.org/10.1590/S1415-65552009000500006 & 20 \\
\hline 10 & A proposição de uma taxonomia para análise da gestão ambiental no Brasil & https://doi.org/10.1590/S1415-65552004000400005 & 20 \\
\hline 11 & Um modelo para o compartilhamento de conhecimento no trabalho & https://doi.org/10.1590/S1415-65552006000200005 & 19 \\
\hline
\end{tabular}


Tabela 2. (Continuação)

\begin{tabular}{lll}
\hline N. & Título \# de & URL para acesso direto ao artigo \\
citaçóes
\end{tabular}

12 Corporate Governance and determinants of capital structure: empirical evidence from Brazilian markets

https://doi.org/10.1590/S1415-65552008000300008

13 Scientific cooperation among graduate programs in the field of business in Brazil: structural evidence in four thematic areas

https://doi.org/10.1590/S1415-65552009000300003

Environmental sustainability: a meta-analysis of production in brazilian management journals

15 Competitiveness models for tourist destinations into the sustainability context

16 Happiness at work: relations with organizational support and social support

17 Entrepreneurial profile and organizational performance

Perfil das pesquisas em contabilidade de custos apresentadas no EnANPAD no período de 1998 a 2002

Avaliação e seleção de fundos de investimentos: um enfoque sobre múltiplos atributos

O processo de internacionalização na ótica da escola nórdica: evidências empíricas em empresas brasileiras

21 Aplicação dos princípios da governança corporativa ao sector público

22 Dynamic Capabilities: What Are They and How to Identify Them?

23 Indicadores de desempenho como direcionadores de valor

24 Pesquisa de satisfação de clientes: o estado-da-arte e proposição de um método brasileiro

25 Ativos intangíveis, ciclo de vida e criação de valor

https://doi.org/10.1590/S1415-65552013000300007

https://doi.org/10.1590/S1415-65552008000300009

https://doi.org/10.1590/S1415-65552010000700005

16

https://doi.org/10.1590/S1415-65552009000300007

https://doi.org/10.1590/S1415-65552007000300009

https://doi.org/10.1590/S1415-65552001000300002

https://doi.org/10.1590/S1415-65552003000100006

https://doi.org/10.1590/S1415-65552007000200002

https://doi.org/10.1590/1982-7849rac20141289

https://doi.org/10.1590/S1415-65552003000100003

https://doi.org/10.1590/S1415-65551998000100007

Consumidores satisfeitos, e então? Analisando a satisfação como antecedente da lealdade

Fatores determinantes da estrutura de capital para empresas latino-americanas

Tecnologias de informação móveis, sem fio e ubíquas: definiçôes, estado-daarte e oportunidades de pesquisa

29 Pesquisa científica da área de marketing: uma revisão histórica

30 The crossroads of organizational learning: a multiparadigmatic view

O discurso e a prática: o que nos dizem os especialistas e o que nos mostram as práticas das empresas sobre os modelos de gestão de recursos humanos

https://doi.org/10.1590/S1415-65552006000300005

https://doi.org/10.1590/S1415-65552004000200004

https://doi.org/10.1590/S1415-65552003000100002

https://doi.org/10.1590/S1415-65552007000400009

https://doi.org/10.1590/S1415-65552006000200010

https://doi.org/10.1590/S1415-65552010000200008

The strategic dimension of the horizontal networks of SMOs: theorizations and evidences

33 Entrepreneurship and economic growth: some empirical evidence

https://doi.org/10.1590/S1415-65552004000500011

Confiança, valor percebido e lealdade em trocas relacionais de serviço: um estudo com usuários de Internet Banking no Brasil

https://doi.org/10.1590/S1415-65552008000400005

https://doi.org/10.1590/S1415-65552005000200008

Interorganizational relationships and results: a study in a network of horizontal cooperation of the central region of Paraná

https://doi.org/10.1590/S1415-65552011000100003

Gestão de pessoas por competências: o caso de uma empresa do setor de telecomunicaçóes

37 A identidade e o contexto organizacional: perspectivas de análise

https://doi.org/10.1590/S1415-65552000000100009

Estratégia, fatores de competitividade e contexto de referência das organizaçóes: uma análise arquetípica

https://doi.org/10.1590/S1415-65552003000500004

https://doi.org/10.1590/S1415-65552002000300002

39 A prática social de gerentes universitários em uma instituição pública

https://doi.org/10.1590/S1415-65552005000300002

Social networks in the scientific production of postgraduate programs of accounting sciences in Brazil

https://doi.org/10.1590/S1415-65552011000100004 
Tabela 2. (Continuação)

\begin{tabular}{|c|c|c|c|}
\hline N.o & Título & URL para acesso direto ao artigo & $\begin{array}{l}\text { \# de } \\
\text { citaçôes }\end{array}$ \\
\hline 42 & Corporate reputation: formative constructs and implications for management & https://doi.org/10.1590/S1415-65552010000200004 & 12 \\
\hline 43 & $\begin{array}{l}\text { Tangibility and intangibility in determining the persistent performance of } \\
\text { Brazilian firms }\end{array}$ & https://doi.org/10.1590/S1415-65552010000500007 & 11 \\
\hline 44 & $\begin{array}{l}\text { Factors that influence the university-industry technology transfer process: the } \\
\text { case of PUCRS }\end{array}$ & https://doi.org/10.1590/S1415-65552012000100005 & 11 \\
\hline 45 & $\begin{array}{l}\text { Proposta para avaliação da gestão do conhecimento em entidade filantrópica: o } \\
\text { caso de uma organização hospitalar }\end{array}$ & https://doi.org/10.1590/S1415-65552003000400009 & 11 \\
\hline 46 & $\begin{array}{l}\text { Modelagem de equações estruturais e satisfação do consumidor: uma } \\
\text { investigação teórica e prática }\end{array}$ & https://doi.org/10.1590/S1415-65552000000300007 & 11 \\
\hline 47 & $\begin{array}{l}\text { The effect of the degree of internationalization on the international competences } \\
\text { and financial performance of Brazilian SMEs }\end{array}$ & https://doi.org/10.1590/S1415-65552012000300007 & 11 \\
\hline 48 & $\begin{array}{l}\text { The Brazilian management graduate program network: analysis of academic } \\
\text { relationships and the attributes of programs }\end{array}$ & https://doi.org/10.1590/S1415-65552009000400004 & 11 \\
\hline 49 & Estrutura de controle das companhias brasileiras de capital aberto & https://doi.org/10.1590/S1415-65552002000100002 & 11 \\
\hline 50 & $\begin{array}{l}\text { Avaliação da satisfação do consumidor utilizando o método de equações } \\
\text { estruturais: um modelo aplicado ao setor elétrico brasileiro }\end{array}$ & https://doi.org/10.1590/S1415-65552004000400002 & 11 \\
\hline
\end{tabular}

Nota. Fonte: Elaborado pelo autor com base em dados gentilmente coletados pelo Prof. Luciano Rossoni na plataforma Web of Science em 3 de março de 2021.

\section{REFERÊNCIAS}

Aguinis, H. \& Solarino, A. M. (2019). Transparency and replicability in qualitative research: The case of interviews with elite informants. Strategic Management Journal, 40(8), 12911315. https://doi.org/10.1002/smj.3015

Aguinis, H., \& Vaschetto, S. J. (2011). Editorial responsibility: Managing the publishing process to do good and do well. Management and Organization Review, 7(3), 407-422. https://doi.org/10.1111/j.1740-8784.2011.00223.x

American Chemical Society. (2017, October 17). Introduction to ACS reviewer lab [Video file]. Video posted to https://www.youtube.com/watch?v=6ZlQmBq-ry4

Arruda Filho, E. J. M., Muylder, C. F., Cançado, A. C., Dholakia, R. R., \& Paladino, A. (2019). Technology perspectives and innovative scenarios applied in the Amazon region. Revista de Administração Contemporânea, 23(5), 607-618. https://doi.org/10.1590/1982-7849rac2019190303

Bandi, F. M., \& Patton, A. J. (2019). Farewell editorial. Journal of Financial Econometrics, 17(3), 339-340. https://doi.org/10.1093/jjfinec/nbz023

Carvalho, J. V. F., Flores, E., \& Valdez, E. A. (2020). Call for papers: Insurance industry. Revista de Administração Contemporânea. Zenodo. http://doi.org/10.5281/zenodo.3900264

Chimenti, P., \& Marques, L. (2020). Teaching Covid-19's Impact on Business. Revista de Administração Contemporânea. Zenodo. http://doi.org/10.5281/zenodo.3744800
Ethiraj, S., Gambardella, A., \& Helfat, C. (2016). Replication in strategic management. Strategic Management Journal, 37(11), 2191-2192. https://doi.org/10.1002/smj.2581

Kimura, H. (2018). Editorial: O aprendizado em editoração científica. Revista de Administração Contemporânea, 22(3), 1-3. https://doi.org/10.1590/1982-7849rac2018180104

Kumar, P., \& Zattoni, A. (2019). Farewell editorial: Exiting editors' perspective on current and future challenges in corporate governance research. Corporate Governance an International Review, 27(1), 2-11. https://doi.org/10.1111/corg.12268

Lanka, E., Lanka, S., Rostron, A., \& Singh, P. (2019). Research methods in qualitative management research. Revista de Administração Contemporânea. Zenodo. http://doi.org/10.5281/zenodo.3934265

Leal, C. C., Oliveira, B., Branco-Illodo, I., \& Salvador, M. L. E. (2020). Call for papers: Nudging and choice architecture. Revista de Administração Contemporânea. Zenodo. http://doi.org/10.5281/zenodo.3900050

Lecompte, M., \& Goetz, J. P. (1982). Problems of reliability and validity in ethnographic research. Review of Educational Research, 52(1), 31-60. https://doi.org/10.3102/00346543052001031

Marques, L., Chimenti, P., \& Mendes-da-Silva, W. (2021). Teaching COVID-19's impact on businesses. Revista de Administração Contemporânea, 25(Spe), e210064. https://doi.org/10.1590/1982-7849rac2021210064.en 
Martins, H. (2019). Tutorial articles: Guiding the practice of quantitative research. Revista de Administração Contemporânea. Zenodo. http://doi.org/10.5281/zenodo.3558928

Martins, H. C. (2020). Tutorial-articles: The importance of data and code sharing. Revista de Administração Contemporânea, 25(1), e200212. https://doi.org/10.1590/1982-7849rac2021200212

Martins, H. C., \& Perlin, M. S. (2020). Call for papers: Data reuse: What new information can we learn from used data? Revista de Administração Contemporânea. Zenodo. https://doi.org/10.5281/zenodo.3858031

Mendes-da-Silva, W. (2018b). Chamada especial governança corporativa de empresas familiares-RAC. Zenodo. https://doi.org/10.5281/zenodo.2247752

Mendes-Da-Silva, W. (2018a). The promotion of transparency and the impact of research on business. Revista de Administraçâo Contemporânea, 22(4), 639-649. https://doi.org/10.1590/1982-7849rac2018180210

Mendes-da-Silva, W. (2018c). Reconhecimento da contribuição do avaliador anônimo. Revista de Administração Contemporânea, 22(5), 1-13. https://doi.org/10.1590/1982-7849rac2018180281

Mendes-da-Silva, W. (2019a). Convergência, comunicação, e impacto da pesquisa em negócios. Revista de Administração Contemporânea, 23(1). https://doi.org/10.1590/1982-7849rac2019180346

Mendes-da-Silva, W. (2019b). Have we been transparent enough? Challenges in replicability and credibility in business research. Revista de Administração Contemporânea, 23(5). https://doi.org/10.1590/1982-7849rac2019190306

Mendes-Da-Silva, W. (2019c). Relatório de gestão da Revista de Administração Contemporânea 2018-2019. Revista de Administração Contemporânea, 23(6), 1-10. https://doi.org/10.1590/1982-7849rac2019190356
Mendes-Da-Silva, W., \& Leal, C. C. (2021). Salami science na era do open data: Déjà lu e accountability na pesquisa em gestão e negócios. Revista de Administração Contemporânea, 25(1), e200194. https://doi.org/10.1590/1982-7849rac2021200194

Mendes-da-Silva, W., Onusic, L. M., \& Giglio, E. M. (2013). Rede de pesquisadores de finanças no Brasil: Um mundo pequeno feito por poucos. Revista de Administração Contemporânea, 17(6), 739-763. https://doi.org/10.1590/S1415-65552013000600007

Moravcsik, A. (2014). Transparency: The revolution in qualitative research. PS: Political Science \& Politics, 47(1), 48-53. https://doi.org/10.1017/S1049096513001789

Oftedal, E., Bertella, G., Grzegorczyk, M., Hill, P. M., \& Lanka, S. (2020). Call for papers: Business models at the crossroad of responsible innovation, sustainability and resilience. Revista de Administração Contemporânea. Zenodo. http://doi.org/10.5281/zenodo.3754339

Plakoyiannaki, E., Wei, T., \& Prashantham, S. (2019). Rethinking qualitative scholarship in emerging markets: Researching, theorizing, and reporting. Management and Organization Review, 15(Spe. 2), 217-234. https://doi.org/10.1017/mor.2019.27

Plos Media (2021, March 16). Thank you for practicing Open Science [Video file]. Video posted to https://youtu.be/Igsi8c4BjI8)

Rossoni, L., \& Rosa, R. A. (2020). Genesis, impact, and identity of the Journal of Contemporary Administration. Revista de Administraçâo Contemporânea, 24(5), 448-473. https://doi.org/10.1590/1982-7849rac2020200126

Tsang, E. W., \& Kwan, K.-M. (1999). Replication and theory development in organizational science: A critical realist perspective. Academy of Management Review, 24(4), 759780. https://doi.org/10.2307/259353 


\section{Autoria}

\section{Wesley Mendes-da-Silva*}

Fundação Getulio Vargas, Escola de Administração de Empresas de São Paulo.

Rua Itapeva, 474, $8^{\circ}$ andar, 01332-000, São Paulo, SP, Brasil.

E-mail: rac.wesley.mendes@gmail.com

(1) https://orcid.org/0000-0002-5500-4872

* Autor Correspondente

\section{Conflito de Interesses}

O autor informou que não há conflito de interesses.

\section{Direitos Autorais}

A RAC detém os direitos autorais deste conteúdo.

\section{Verificação de Plágio}

A RAC mantém a prática de submeter todos os documentos aprovados para publicaçẫo à verificação de plágio, mediante o emprego de ferramentas específicas, e.g.: iThenticate.

\section{CORPO EDITORIAL CIENTÍFICO E EQUIPE EDITORIAL PARA ESTA EDIÇÃO:}

\section{Conselho Editorial}

Anielson Barbosa da Silva (UFPB, João Pessoa, Brasil)

Antonio Carlos Gastaud Maçada (UFRGS, Porto Alegre, Brasil)

Ely Laureano Paiva (FGV, São Paulo, Brasil)

Rogério Hermida Quintella (NPGA/UFBA, Salvador, Brasil)

Valmir Emil Hoffmann (UnB, Brasília, Brasil)

Wesley Mendes-da-Silva (FGV/ EAESP, São Paulo, Brasil)

\section{Editor-chefe}

Wesley Mendes-da-Silva (FGV/ EAESP, São Paulo, Brasil)

\section{Editores Associados}

Eduardo da Silva Flores (FEA/USP,, São Paulo, Brasil)

Emílio José M. Arruda Filho (UNAMA, Belém, Brasil)

Fabio Caldieraro (EBAPE/FGV, São Paulo, Brasil)

Gilnei Luiz de Moura (UFSM, Santa Maria, Brasil)

Henrique Castro Martins (IAG PUC-Rio, Rio de Janeiro, Brasil)

Ismael Ali Ali (Kent State University, Ohio, EUA)

Marcus Cunha Junior (University of Georgia, EUA)
Paula Castro Pires de Souza Chimenti (UFRJ/Coppead, Rio de Janeiro, Brasil)

Paulo César Matui (UniGranRio, Rio de Janeiro, Brasil)

\section{Corpo Editorial Científico}

André Luiz Maranhão de Souza-Leão (UFPE, Recife, Brasil)

Aureliano Angel Bressan (CEPEAD/UFMG, Belo Horizonte, Brasil)

Bryan Husted (York University, Canadá)

Carlos M. Rodriguez (Delaware State University, EUA)

Cristiana Cerqueira Leal (Universidade do Minho, Portugal)

Diógenes de Souza Bido (Mackenzie, São Paulo, Brasil)

Erica Piros Kovacs (Kelley School of Business/Indiana University, EUA)

Elin Merethe Oftedal (University of Stavanger, Noruega)

Fábio Frezatti (FEA/USP, São Paulo, Brasil)

Felipe Monteiro (INSEAD Business School, EUA)

Howard J. Rush (University of Brighton, Reino Unido)

James Robert Moon Junior (Georgia Institute of Technology, EUA)

John L. Campbell (University of Georgia, EUA)

José Antônio Puppim de Oliveira (United Nations University, Yokohama, Japão)

Julián Cárdenas (Freie Universität, Berlin, Alemanha)

Lucas A. B. de Campos Barros (FEA/USP, São Paulo, Brasil)

Luciano Rossoni (UniGranRio, Rio de Janeiro, Brasil)

M. Philippe Protin (Université Grenoble Alpes, França)

Paulo Estevão Cruvinel (Embrapa Instrumentaçáo, São Carlos, Brasil)

Rodrigo Bandeira de Mello (Merrimack College, EUA)

Rodrigo Verdi (MIT Massachusetts Institute of Technology, Cambridge, EUA)

Valter Afonso Vieira, (UEM, Maringá, Brasil)

Wagner Kamakura (Jones Graduate School of Business, Rice University, Houston, EUA)

\section{Editoração}

Diagramação e normas da APA: Kler Godoy (ANPAD, Maringá, Brasil); Simone L. L. Rafael (ANPAD, Maringá, Brasil).

Periodicidade: Publicação contínua.

Circulação: Acesso totalmente gratuito.

\section{Indexadores e Diretórios}

Scielo, Redalyc, DOAI, Latindex, Cengage/GALE, Econpapers, EBSCO, MIAR, Proquest, SPELL, Cabell's, Ulrichs, CLASE, DIADORIM, ERIHPlus, EZB， EuroPub， OasisBR, WorldWideScience, Google Scholar, Capes/Qualis, Citefactor.org, Index Copernicus International, Sherpa Romeo. 